\title{
Functional Outcomes of Arthroscopic Combined Anterior Cruciate Ligament Reconstruction and Meniscal Repair: A Retrospective Analysis
}

\author{
Shirish Pathak, M.S., Abhinav Bharadwaj, M.S., Prateek Patil, M.S., Sudarshan Raut, M.S., \\ and Srikanth RV, M.S.
}

\begin{abstract}
Purpose: To evaluate the outcomes of arthroscopic meniscal repair performed in combination with anterior cruciate ligament (ACL) repair. Methods: This study presents a case series of 34 patients who underwent repair of meniscal tears along with ACL reconstruction from 2014 to 2016. Cases of discoid meniscal lesions and combined or ligament injuries other than ACL injuries were excluded. Patients were followed up periodically, at 3, 6, 9, 12, and 24 months. Preoperative and postoperative functional evaluations were performed using visual analog scale, International Knee Documentation Committee, and Lysholm knee scores. Results: The mean age of the patients was 29.1 years (range, 17-44 years). The mean follow-up period was $18 \pm 7.8$ months (range, 6-24 months). Among the 34 individual knees, 1 patient (3\%) underwent both medial and lateral meniscal repairs. Medial meniscal repair was performed in $20 \mathrm{knees} \mathrm{(59 \% ),} \mathrm{whereas}$ the lateral meniscus was repaired in 13 knees (38\%). A longitudinal tear was the most common type of tear pattern, followed by radial (6 patients) and complex ( 3 patients) tear patterns. The radial and complex tears were treated with an additional partial meniscectomy. The mean International Knee Documentation Committee score was 38.46 preoperatively and improved to 80.30 at final postoperative follow-up (statistically significant difference, $P<.01$ ). The mean Lysholm score was 50.30 preoperatively and improved to 91.40 at final postoperative follow-up (statistically significant difference, $P<.01)$. According to the Lysholm knee score, 31 patients $(89 \%)$ had excellent or good results. The mean visual analog scale score decreased from 7.3 preoperatively to 2 postoperatively. The clinical success rate of the repairs was $89 \%$. Of 35 repairs, 4 (11\%) had retears. Conclusions: Arthroscopic meniscal repair along with ACL reconstruction provided predictable high rates of meniscal healing and yielded favorable functional and clinical results. Patient selection remains one of the most important prognostic factors. Level of Evidence: Level IV, therapeutic case series.
\end{abstract}

C ombined injuries to the menisci and anterior cruciate ligament (ACL) are frequently seen in the acutely injured knee. ${ }^{1}$ Historically, it was believed that the menisci served no functional purpose, and they were often excised with open total meniscectomy. In 1948, Fairbank ${ }^{2}$ reported the clinical outcomes of 107

From the Sports Injuries Department, Deenanath Mangeshkar Hospital and Research Centre, Pune, India.

The authors report that they have no conflicts of interest in the authorship and publication of this article. Full ICMJE author disclosure forms are available for this article online, as supplementary material.

Received July 15, 2019; accepted November 4, 2019.

Address correspondence to Prateek Patil, M.S., Department of Shoulder and Sports Injuries, First Floor, Superspeciality Building, Deenanath Mangeshkar Hospital and Research Centre, Erandwane, Pune, 411004, Maharashtra, India.E-mail:patilcprateek@gmail.com

(C) 2019 THE AUTHORS. Published by Elsevier Inc. on behalf of the Arthroscopy Association of North America. This is an open access article under the CC BY-NC-ND license (http://creativecommons.org/licenses/by-nc-nd/4.0/). 2666-061X/19848

https://doi.org/10.1016/j.asmr.2019.11.001 patients after total meniscectomy and found that most had progressive flattening of the condyle, narrowing of the joint space, and ridge formation. This landmark study significantly changed the approach to dealing with meniscal tears. The accumulation of evidence of long-term consequences and altered biomechanics in the knee after meniscectomy has placed greater emphasis on meniscus-preserving techniques.

Several studies have shown that meniscal repair results in fewer degenerative changes than partial or subtotal meniscectomy. ${ }^{3-5}$ The results of meniscal repair along with ACL reconstruction have shown better healing and lower retear rates compared with isolated meniscal repairs.

The purpose of this study was to evaluate the outcomes of arthroscopic meniscal repair performed in combination with ACL repair. We hypothesized that meniscal repair along with ACL reconstruction would result in significantly higher healing rates and improved postoperative functional outcome scores. 


\section{Methods}

From 2014 to 2016, patients who underwent repair of meniscal tears along with ACL reconstruction were identified. The inclusion criteria were surgery within 3 months of injury, tears located in the red-red or redwhite zone, and good tissue quality. The exclusion criteria were discoid meniscal lesions and combined ligament injuries other than ACL injuries.

All surgical procedures were performed under tourniquet control. All ACL reconstructions used quadrupled hamstring tendon (semitendinosus or semitendinosus-gracilis) autograft. Meniscal repair was performed primarily using an all-inside technique, whereas outside-in and inside-out techniques were used alone or in conjunction with the all-inside technique.

The postoperative protocol consisted of range-ofmotion exercises from $0^{\circ}$ to $90^{\circ}$ and non-weightbearing walking with a posterior long knee brace for 3 weeks after surgery. This was followed by gradual progression to full range of motion and partial weight bearing. Full weight bearing began at 4 to 5 weeks after surgery. Running and preinjury activities were permitted at 5 to 6 months after surgery, depending on strength and neuromuscular coordination.

Postoperatively, patients were followed up periodically, at 3, 6, 9, 12, and 24 months. Preoperative and postoperative functional evaluations were performed using visual analog scale (VAS), International Knee Documentation Committee (IKDC), and Lysholm knee scores. Patients with a minimum of 6 months' followup were included in the study. The criteria for clinical success included a negative McMurray test finding and absence of joint-line tenderness, locking, and swelling. Diagnosis of a failed repair was based on clinical symptoms and signs suggestive of a retear of the repaired meniscus. When a retear was suspected clinically, the status of healing at the repair site was assessed with 3-T magnetic resonance imaging (MRI) followed by repeated arthroscopy. Conventional MRI is useful in diagnosing extrameniscal pathology postoperatively in patients. However, it has a lower sensitivity for detecting retears after partial meniscectomy because it relies primarily on showing fluid entering the meniscal retear on T2-weighted images. Conventional MRI is also less accurate after meniscal repair, in which the repair site usually maintains an altered signal for years. Magnetic resonance arthrography has a higher sensitivity for detecting retears in menisci and is the procedure of choice in many situations. Because imaging investigations are expensive and not without risk to the joint, only symptomatic patients were subjected to postoperative MRI. Asymptomatic retears might be missed using this protocol but unnecessary MRI scans would be avoided. ${ }^{6,7}$
The study results were tested for significance using the Wilcoxon signed rank test. Because the sample size was small, a normal distribution of the data cannot be assumed. Hence, a nonparametric test for comparison of paired data (Wilcoxon rank ligand test) was used to test the significance of the results.

\section{Results}

During the study period from 2014 to 2016,445 patients underwent ACL reconstructions at our center. All ACL reconstructions were performed using a quadrupled hamstring tendon (semitendinosus or semitendinosus-gracilis) autograft. The autograft was fixed using a variable-loop suspensory fixation device on the femoral side and an interference screw or a suture disc on the tibial side. Among these patients, 225 $(50.56 \%)$ had concomitant meniscal tears. Of these patients with concomitant meniscal tears, 34 (15.11\% of the patients with ACL and concomitant meniscal tears) underwent meniscal repair with combined ACL reconstruction. The remaining 191 patients $(84.89 \%$ of the patients with concomitant meniscal tears) were identified to have irreparable meniscal tears and underwent partial meniscectomy instead. The reason for irreparability of the meniscal tear was either the tear pattern, size, or location or the quality of the remnant meniscal tissue.

The mean age of the patients was 29.1 years (range, 17-44 years). Among the 35 menisci that were repaired, 21 patients had a follow-up period of 24 months, 7 had a follow-up period of 12 months, and 7 had a follow-up period of 6 months. The mean follow-up period was 18 \pm 7.8 months (range, 6-24 months). Among the 34 individual knees, 1 patient $(3 \%)$ underwent both medial and lateral meniscal repairs. Medial meniscal repair was performed in 20 knees (59\%), whereas the lateral meniscus was repaired in 13 knees $(38 \%)$ (Fig 1). Only concomitant longitudinal meniscal tears involving the outer and middle third of the meniscus were repaired. Six patients had a radial tear in the adjacent meniscus that was treated with an additional partial meniscectomy. A complex tear pattern was seen in 3 patients and was treated with an additional partial meniscectomy. The case series also included 1 buckethandle tear and 3 longitudinal tears in the posterior horn that were treated with partial meniscectomy and underwent a repair of the adjacent meniscus in the same knee.

An all-inside technique alone was used to treat most of the medial meniscal tears (14 [64\%]), followed by a combination of all-inside and outside-in techniques ( 7 [31\%]); 1 tear (5\%) was repaired with a combination of inside-out, outside-in, and all-inside techniques. In the lateral meniscus, 9 tears $(69 \%)$ were treated by an all-inside technique alone, whereas 2 tears were treated 


\section{Meniscus involved in study subjects}

Fig 1. Meniscus involved in study subjects, displayed as n $(\%)$.

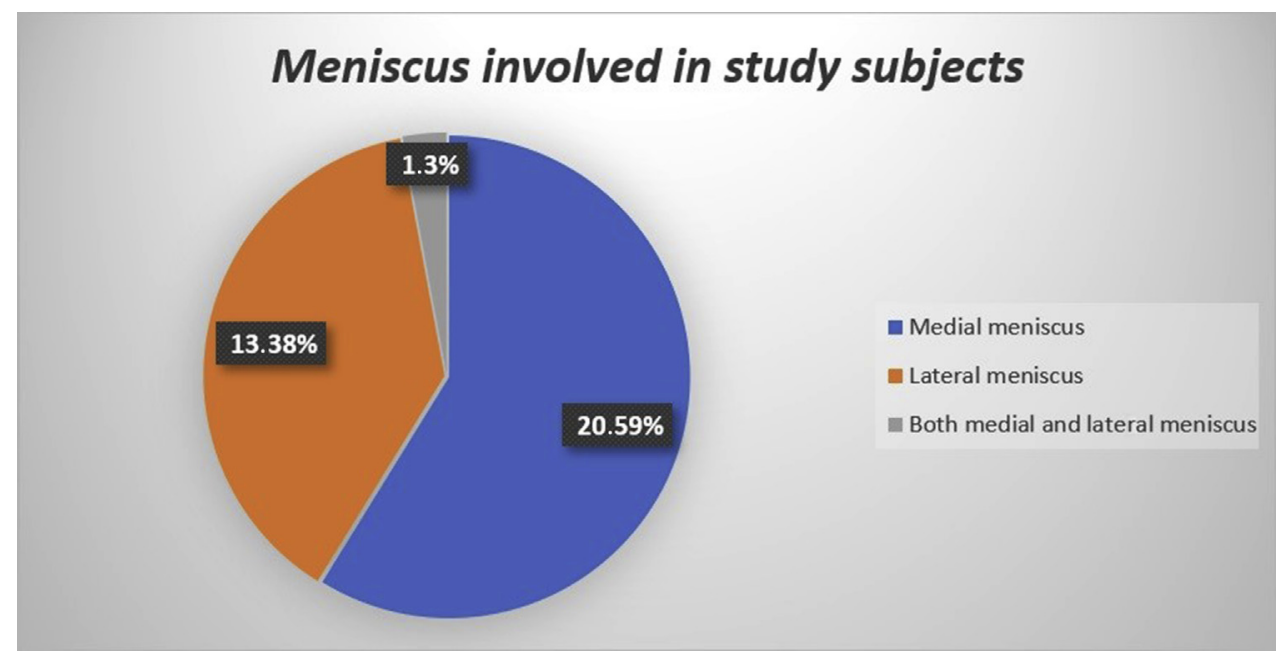

by a combination of all-inside and outside-in techniques and 2 tears were repaired by the outside-in technique alone (Fig 2).

Among the 35 meniscal repairs, 33 (94.3\%) were performed using an all-inside repair implant. Only 2 repairs were completed without the use of the all-inside repair implant. Of the 33 repairs that underwent an allinside repair with or without an additional repair technique, $24(72.72 \%)$ were performed using 2 allinside repair implants, $6(18.18 \%)$ were performed using a single all-inside repair implant, and $3(9.1 \%)$ were completed using 3 all-inside repair implants.

Among the 35 repairs, 12 (34.3\%) involved the use of the outside-in technique with or without an additional technique ( 8 in the medial meniscus and 4 in the lateral meniscus). Outside-in repair involved the use of 4 to 5 stitches with non-absorbable braided polyester suture material. Only 1 repair $(2.85 \%)$ involved the use of the inside-out technique with an additional technique. This was completed using 3 stitches with non-absorbable braided polyester suture material.

The mean IKDC score was 38.46 preoperatively and improved to 80.30 at final postoperative follow-up (statistically significant difference, $P=.00008$ ). The mean Lysholm score was 50.30 preoperatively and improved to 91.40 at final postoperative follow-up (statistically significant difference, $P=.00008$ ). According to the Lysholm knee score, 31 patients (89\%) had excellent or good results. The mean VAS score decreased significantly from 7.3 preoperatively to 2 postoperatively $(P<.00001)$ (Fig 3$)$.

The clinical success rate of the repairs was $89 \%$. Of 35 repairs, $4(11 \%)$ had retears. All the retears were observed in the medial meniscus. In all cases of retear, recurrence of meniscal symptoms was noted and the location of the retear involved the original tear site. Three retears were diagnosed within 6 months after the operation. One was diagnosed at 18 months. All retears were bucket handle-type tears in the red-white zone. Two patients were treated by partial meniscectomy because of the unacceptable symptoms or mechanical nature of the symptoms. Two patients opted for a conservative line of management. Because of the small number, it is difficult to recommend an optimal line of management in symptomatic retears.

\section{Discussion}

This study showed significant improvements in mean IKDC (from 38.46 to 80.30), Lysholm (from 50.30 to 91.40), and VAS (from 7.3 to 2) scores after combined ACL and meniscal repair. The overall success rate of the procedure was $89 \%$, with an $11 \%$ retear rate in the meniscus.

Around $40 \%$ to $65 \%$ of patients with ACL injuries have concomitant meniscal injuries. ${ }^{1,8}$ The association of ACL injury and ACL reconstruction with early posttraumatic osteoarthritis has been well established. ${ }^{9,10}$ There has been an increased emphasis on repair of the meniscus over the past 3 decades because this structure is essential in preventing osteoarthritis. ${ }^{11}$

Knees treated with meniscectomy have been shown to evolve into osteoarthritic knees 3 to 7 times more commonly than osteoarthritis occurring in normal knees. $^{12}$ The association of an ACL lesion with a meniscal tear increases the prevalence of long-term radiographic osteoarthritis (prevalence of 59\%) compared with ACL injury alone (prevalence of $31 \%) .{ }^{13}$ Shelbourne et al. ${ }^{14}$ concluded from their study that further follow-up was needed to recommend definitive treatment but their meniscectomy group had significantly more pain compared with their meniscal repair group. No statistically significant difference was found between groups for the subjective subscores for swelling, stability, and activity level. ${ }^{14}$ 


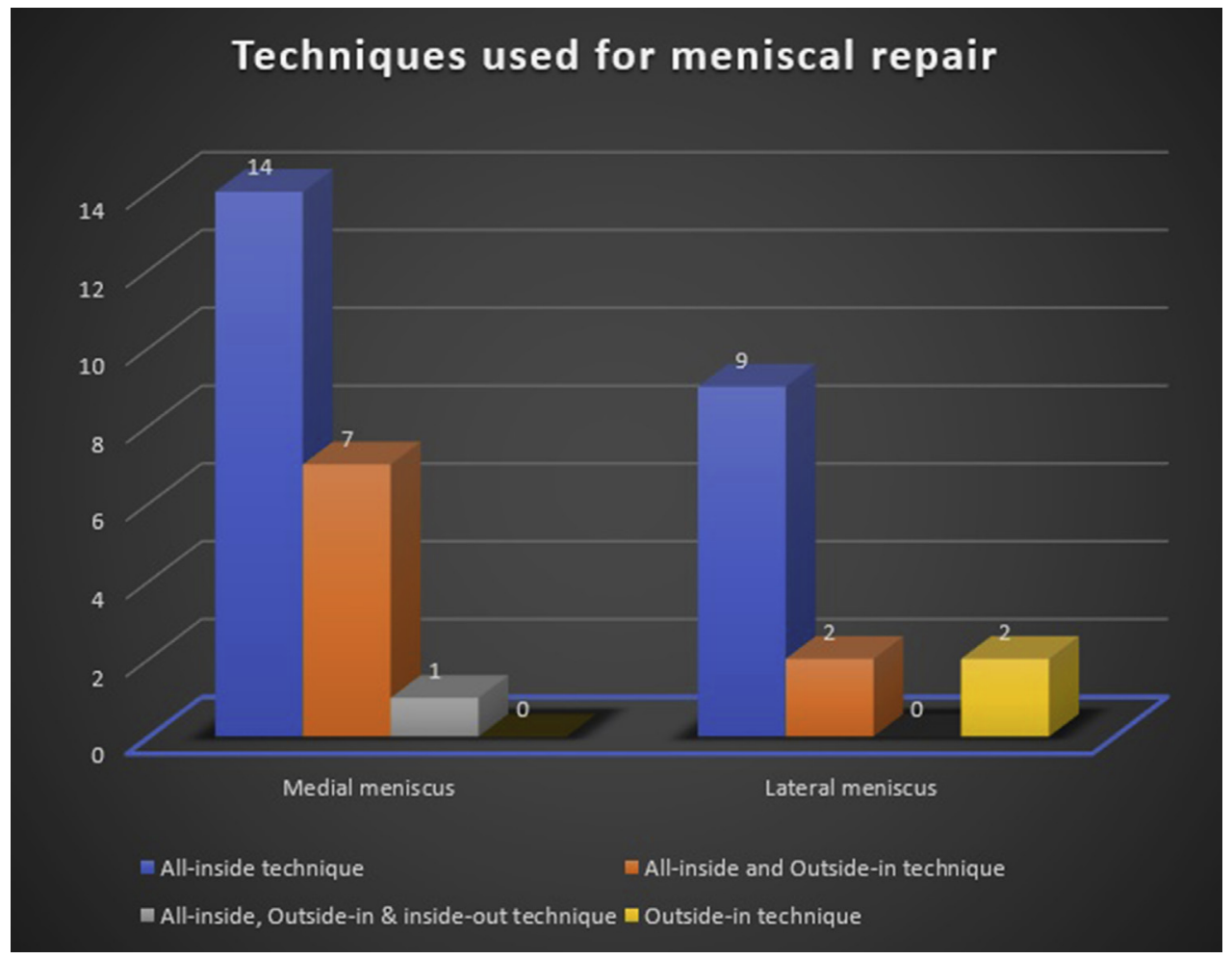

Fig 2. Techniques used for meniscal repair.

The meniscus plays an important role in both knee stability and load distribution. ${ }^{15}$ It has been shown that meniscectomy plays a role in knee instability, and some authors have suggested a risk of ACL graft failure in patients undergoing simultaneous meniscectomy (after the removal of the posterior horn). ${ }^{16}$

Papageorgiou et al. ${ }^{17}$ performed a biomechanical study on cadaveric knees and found that after medial meniscectomy, the forces on the ACL graft increased by between $33 \%$ and $50 \%$. They suggested that ACL grafts may be at increased risk of failure in the absence of the medial meniscus. Thus, meniscal repair may protect the ACL graft from increasing stress forces.

Arno et al. ${ }^{18}$ showed in their cadaveric study that partial medial meniscectomy, in which resection exceeded $46 \%$ of the original width of the medial
Fig 3. Functional score assessment. (IKDC, International Knee Documentation Committee; VAS, visual analog scale.)

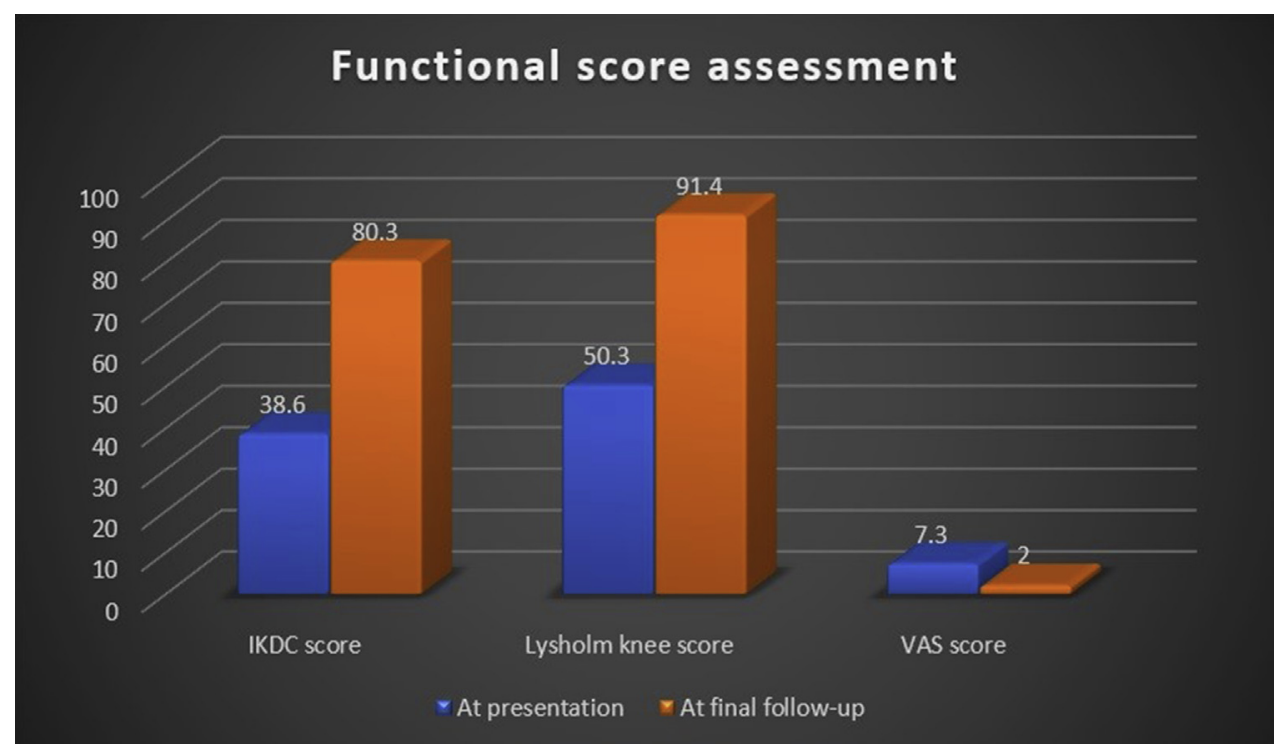


meniscus, altered the anteroposterior stability of the medial femoral condyle and led to increased anteroposterior laxity. This may lead to abnormal cartilage loads and early osteoarthritis.

Various studies comparing the results of meniscal repair with partial meniscectomy have shown better functional and radiologic results after meniscal repair, with failure rates of repair ranging from $10 \%$ to $35 \% .{ }^{15,18,19}$ A systematic review of studies reporting the outcomes of meniscal repair at a minimum of 5 years postoperatively by Nepple et al. ${ }^{19}$ showed a similar meniscal failure rate of $22.3 \%$ to $24.3 \%$ for all techniques investigated.

In our study, the failure rate of meniscal repair was $11 \%$. This finding may be a result of the relatively short follow-up period. Meniscal repairs performed concomitantly with ACL reconstruction have been shown to have better outcomes than isolated meniscal repairs. In patients where a retear was suspected based on clinical symptoms, a 3-T MRI scan of the involved knee was performed. MRI characteristics of the postoperative meniscus that can be used to identify meniscal pathology are as follows: contour (smooth or irregular), T2 line through the meniscus (no line, intermediate signal line, intermediate to high signal line, and high fluid-like signal line), displaced meniscal fragment, and change in the signal pattern through the meniscus compared with baseline MRI. Menisci with no T2 signal line were not torn at surgery. The most useful MRI characteristics to predict torn postoperative menisci at surgery were a change in the signal pattern through the meniscus compared with baseline MRI and a displaced meniscal fragment followed by a high $\mathrm{T} 2$ line through the meniscus, an intermediate to high $\mathrm{T} 2$ line through the meniscus, and an irregular meniscal contour. ${ }^{20}$ On the basis of these MRI characteristics, Kijowski et al. ${ }^{20}$ reported 64 meniscal retears $(42.25 \%)$ among 148 patients.

Matsushita et al. ${ }^{21}$ reported good results for isolated meniscal healing after repair. They found complete meniscal healing in 49 knees $(56 \%)$, incomplete meniscal healing in 19 knees $(22 \%)$, and retears in 19 knees $(22 \%)$. The literature reports an incidence of retears ranging from $20 \%$ to almost $40 \%$. The risk factors for meniscal retears were the size (length of the tear), the presence of a complete tear, and a positive pivot-shift test finding (residual instability). ${ }^{21}$

A systematic review by Paxton et al. ${ }^{22}$ showed an overall reoperation rate of $24 \%$ after meniscal repair compared with $14 \%$ when performed in conjunction with ACL reconstruction, and this relation was maintained even when analyzed by specific repair methods and devices. Comparison of reoperation rates and clinical outcomes of meniscal repair versus meniscectomy at short-term and long-term follow-up showed lower reoperation rates (1.4\% and 3.9\%, respectively) for partial meniscectomy than for isolated meniscal repair (16.5\% and 20.7\%, respectively). Paxton et al. concluded that although meniscal repairs have higher reoperation rates than partial meniscectomies, they have better long-term outcomes.

In a study of reoperations after meniscal repair, with and without concomitant ACL reconstruction, Wasserstein et al. ${ }^{23}$ concluded that meniscal repair performed in conjunction with ACL reconstruction carries a 7\% absolute and $42 \%$ relative risk reduction of reoperation after 2 years compared with isolated meniscal repair. The potential explanation for the better results could be the abundance of blood and growth factors brought into the knee by drilling bony tunnels, relatively limited patient activity, and less aggressive rehabilitation after combined procedures, as well as better tissue quality of the meniscus at the time of repair.

A study by Lester et al. ${ }^{24}$ analyzing the costeffectiveness of meniscectomy versus meniscal repair with combined ACL reconstruction recently concluded that the findings favored the meniscal repair group. They reported a higher failure rate in the meniscal repair group ( $10 \%$ vs $4 \%)$, but the overall rate of arthritis (symptomatic plus asymptomatic) was much higher in the meniscectomy group $(34.5 \%$ vs $6.5 \%)$.

In our study, all retears occurred in the medial meniscus. Of the 4 retears, 3 occurred within 6 months and 1 occurred after 1.5 years. Several studies have previously suggested a higher failure rate for medial meniscal repair than for lateral meniscal repair, with medial repairs failing earlier than lateral repairs. ${ }^{22,25,26}$ This may be due to the fact that the medial side of the meniscus is anchored more tightly to the tibial plateau and the fact that the medial compartment bears higher biomechanical loads. ${ }^{17,18}$ If there is residual laxity after ACL reconstruction, the medial meniscus may encounter greater stress because it is a secondary stabilizer to anterior tibial translation.

Noyes and Barber-Westin ${ }^{26}$ performed a systematic review to determine the incidence of meniscectomy, meniscal repair, and meniscal tears left in situ during ACL reconstruction. They included 11,711 meniscal tears, of which $65 \%$ (7,621 tears) were treated by meniscectomy, 26\% (3,022 tears) were treated by repair, and $9 \%$ (1,068 tears) were left in situ. They concluded that meniscectomy is performed 2 to 3 times more frequently than meniscal repair during ACL reconstruction. The number of potentially repairable meniscal tears that were treated by resection in this systematic review could not be identified. This is concerning because studies have shown that regardless of knee stability obtained after ACL reconstruction, meniscectomy accelerates degenerative joint changes.

\section{Limitations}

The limitations of this study include the small sample size $(\mathrm{N}=35)$ and the relatively short follow-up period. 
In addition, the diagnosis of failure was based on clinical evaluation alone, with MRI being performed only in patients who experienced symptoms of failure of the meniscal repair. Therefore, asymptomatic repair failure was not included among the failed cases.

\section{Conclusions}

Arthroscopic meniscal repair along with ACL reconstruction provided predictably high rates of meniscal healing and yielded favorable functional and clinical results. Patient selection remains one of the most important prognostic factors.

\section{References}

1. Smith JP, Barrett GR. Medial and lateral meniscal tear patterns in anterior cruciate ligament-deficient knees. A prospective analysis of 575 tears. Am J Sports Med 200 1;29: 415-419.

2. Fairbank TJ. Knee joint changes after meniscectomy. J Bone Joint Surg Br 1948;30B:664-670.

3. Xu C, Zhao J. A meta-analysis comparing meniscal repair with meniscectomy in the treatment of meniscal tears: The more meniscus, the better outcome? Knee Surg Sports Traumatol Arthrosc 2015;23:164-170.

4. Lutz C, Dalmay F, Ehkirch FP, et al. Meniscectomy versus meniscal repair: 10 Years radiological and clinical results in vertical lesions in stable knee. Orthop Traumatol Surg Res 2015;101:S327-S331 (suppl).

5. McDermott ID, Amis AA. The consequences of meniscectomy. J Bone Joint Surg Br 2006;88:1549-1556.

6. Davis KW, Tuite MJ. MR imaging of the postoperative meniscus of the knee. Semin Musculoskelet Radiol 2002;6: 35-45.

7. Baker JC, Friedman MV, Rubin DA. Imaging the postoperative knee meniscus: An evidence-based review. AJR Am J Roentgenol 2018;211:519-527.

8. Levy AS, Meier SW. Approach to cartilage injury in the anterior cruciate ligament-deficient knee. Orthop Clin North Am 2003;34:149-167.

9. Lohmander LS, Ostenberg A, Englund M, Roos H. High prevalence of knee osteoarthritis, pain, and functional limitations in female soccer players twelve years after anterior cruciate ligament injury. Arthritis Rheum 2004;50:3145-3152.

10. von Porat A, Roos E, Roos H. High prevalence of osteoarthritis 14 years after an anterior cruciate ligament tear in male soccer players: A study of radiographic and patient relevant outcomes. Ann Rheumatol Dis 2004;63:269-273.

11. Stein T, Mehling AP, Welsch F, von Eisenhart-Rothe R, Jäger A. Long-term outcome after arthroscopic meniscal repair versus arthroscopic partial meniscectomy for traumatic meniscal tears. Am J Sports Med 2010;38:1542-1548.

12. Ahlbäck S. Osteoarthrosis of the knee. A radiographic investigation. Acta Radiologica: Diagnosis (Stockh) 1968; 277 (suppl):7-72.
13. Deledda D, Rosso F, Cottino U, Bonasia DE, Rossi R. Results of meniscectomy and meniscal repair in anterior cruciate ligament reconstruction. Joints 2016;3: $151-157$.

14. Shelbourne KD, Dersam MD. Comparison of partial meniscectomy versus meniscus repair for bucket-handle lateral meniscus tears in anterior cruciate ligament reconstructed knees. Arthroscopy 2004;20:581-585.

15. Radin EL, de Lamotte F, Maquet P. Role of the menisci in the distribution of stress in the knee. Clin Orthop Relat Res 1984;185:290-294.

16. Yammine K. Effect of partial medial meniscectomy on anterior tibial translation in stable knees: A prospective controlled study on 32 patients. BMC Sports Sci Med Rehabil 2013;5:17.

17. Papageorgiou CD, Gil JE, Kanamori A, Fenwick JA, Woo SL, Fu FH. The biomechanical interdependence between the anterior cruciate ligament replacement graft and the medial meniscus. Am J Sports Med 2001;29: 226-231.

18. Arno S, Bell CP, Uquillas C, Borukhov I, Walker PS. Tibiofemoral contact mechanics following a horizontal cleavage lesion in the posterior horn of the medial meniscus. J Orthop Res 2015;33:584-590.

19. Nepple JJ, Dunn WR, Wright RW. Meniscal repair outcomes at greater than five years: A systematic literature review and meta-analysis. J Bone Joint Surg Am 2012;94: 2222-2227.

20. Kijowski R, Rosas H, Williams A, Liu F. MRI characteristics of torn and untorn post-operative menisci. Skeletal Radiol 2017;46:1353-1360.

21. Matsushita T, Nagai K, Araki D, et al. Factors associated with the status of meniscal tears following meniscal repair concomitant with anterior cruciate ligament reconstruction. Connect Tissue Res 2017;58:386-392.

22. Paxton ES, Stock MV, Brophy RH. Meniscal repair versus partial meniscectomy: A systematic review comparing reoperation rates and clinical outcomes. Arthroscopy 2011;27:1275-1288.

23. Wasserstein D, Dwyer T, Gandhi R, Austin PC, Mahomed N, Ogilvie-Harris D. A matched-cohort population study of reoperation after meniscal repair with and without concomitant anterior cruciate ligament reconstruction. Am J Sports Med 2013;41:349-355.

24. Lester JD, Gorbaty JD, Odum SM, Rogers ME, Fleischli JE. The cost-effectiveness of meniscal repair versus partial meniscectomy in the setting of anterior cruciate ligament reconstruction. Arthroscopy 2018;34:2614-2620.

25. Westermann RW, Wright RW, Spindler KP, Huston LJ, MOON Knee Group, Wolf BR. Meniscal repair with concurrent anterior cruciate ligament reconstruction: Operative success and patient outcomes at 6-year followup. Am J Sports Med 2014;42:2184-2192.

26. Noyes FR, Barber-Westin SD. Treatment of meniscus tears during anterior cruciate ligament reconstruction. Arthroscopy 2012;28:123-130. 